\title{
Short early filling deceleration time on day 1 after acute myocardial infarction is associated with short and long term left ventricular remodelling
}

\author{
P Otašević, A N Nešković, Z Popović, A Vlahović, D Bojić, M Bojić, A D Popović
}

\begin{abstract}
Objective-To assess the relations between early filling deceleration time, left ventricular remodelling, and cardiac mortality in an unselected group of postinfarction patients.

Design and patients-Prospective evaluation of 131 consecutive patients with first acute myocardial infarction. Echocardiography was performed on day 1, day 2, day 3, day 7, at three and six weeks, and at three, six, and 12 months after infarction. According to deceleration time on day 1 , patients were divided into groups with short $(<150 \mathrm{~ms})$ and normal deceleration time ( $\geqslant 150 \mathrm{~ms}$ ).

Setting-Tertiary care centre.

Results-Patients with a short deceleration time had higher end systolic and end diastolic volume indices and a higher wall motion score index, but a lower ejection fraction, in the year after infarction. These patients also showed a significant increase in end diastolic $(p<0.001)$ and end systolic volume indices $(p=0.007)$ during the follow up period, while ejection fraction and wall motion score index remained unchanged. In the group with normal deceleration time, end diastolic volume index increased $(p<0.001)$ but end systolic volume index did not change; in addition, the ejection fraction increased $(\mathrm{p}=0.002)$ and the wall motion score index decreased $(p<0.001)$. One year and five year survival analysis showed greater cardiac mortality in patients with a short deceleration time $(\mathrm{p}=0.04$ and $\mathrm{p}=0.02$, respectively). In a Cox model, which included initial ejection fraction, infarct location, and infarct size, deceleration time on day 1 was the only significant predictor of five year mortality.

Conclusions-A short deceleration time on day 1 after acute myocardial infarction can identify patients who are likely to undergo left ventricular remodelling in the following year. These patients have a higher one year and five year cardiac mortality.

(Heart 2001;85:527-532)
\end{abstract}

Keywords: deceleration time; left ventricular remodelling; acute myocardial infarction

Left ventricular remodelling following acute myocardial infarction is a well known phenomenon, ${ }^{1}$ occurring in the earliest postinfarction phase and continuing for weeks or months. ${ }^{2}$ It has been shown that left ventricular volume and ejection fraction are major predictors of prognosis in these patients. ${ }^{3}$ However, the prognostic value of left ventricular filling indices in patients with acute myocardial infarction is less well defined. It has recently been suggested that a restrictive transmitral filling pattern-as a marker of diastolic dysfunction-provides significant additional prognostic information in such patients. ${ }^{4}$ The usual definition of restrictive filling includes the ratio of maximum early to atrial (E:A) filling velocities. This variable can be influenced by heart rate, left ventricular systolic function, and loading conditions, ${ }^{5}$ making its interpretation in particular patients difficult. On the other hand, it appears that the early filling deceleration time is largely independent of these factors ${ }^{5}$ and reliably reflects left ventricular chamber stiffness, ${ }^{6}$ suggesting that this single variable may provide similar prognostic information. In addition, deceleration time is inversely correlated with pulmonary capillary wedge pressure, which is one of the strongest predictors of mortality after infarction. ${ }^{78}$ It has recently been shown that deceleration time was the best single predictor of all cause mortality in both symptomatic and asymptomatic patients with left ventricular dysfunction. ${ }^{9}$ However, the relation between left ventricular transmitral filling pattern early after infarction and subsequent left ventricular dilatation has not been fully clarified.

Our aim in this study was to evaluate the relation between early filling deceleration time and left ventricular remodelling in the year following a first acute myocardial infarct, and to assess the impact of a short early filling deceleration time on one year and five year cardiac mortality.

\section{Methods}

STUDY PATIENTS

We prospectively evaluated 131 consecutive patients with a first acute myocardial infarct who met the following criteria: age $\leqslant 70$ years; chest pain lasting more than 30 minutes; ST segment elevation $\geqslant 2 \mathrm{~mm}$ at least in two ECG leads; transient elevation of creatine kinase and/or MB isoenzyme; and an echocardiogram performed within 24 hours of the onset of pain.

\section{TREATMENT}

Thrombolytic treatment (intravenous streptokinase 1500000 units over 30-60 minutes) was given in 81 patients; the remaining 50 
patients were not treated with thrombolysis at the discretion of their attending cardiologist, because they were admitted late after the onset of pain or had contraindications to the treatment. The use of thrombolysis, nitrates, $\beta$ blockers, calcium channel blockers, angiotensin converting enzyme (ACE) inhibitors, diuretics, and digoxin was similar in patients with and without restrictive filling.

\section{ECHOCARDIOGRAMS}

Echocardiographic examinations were performed in the following sequence: day 1 , day 2 , day 3 , day 7 , at three and six weeks, and at three, six, and 12 months after the index infarction. All examinations were performed with an Acuson 128 machine (Mountain View, California, USA), using a $2.5 \mathrm{MHz}$ transducer, and stored on videotape for later analysis.

Transmitral flow recordings were obtained from the apical four chamber view, with the sample volume positioned at the tips of the mitral leaflets. Modal flow velocities were traced in three consecutive beats after quiet expiration and averaged. As tachycardia alters diastolic filling and leads to the fusion of the early and atrial filling waves, studies in which heart rate was $>100$ beats $/ \mathrm{min}$ were not included in the analysis. We also excluded studies in which atrial fibrillation or significant mitral regurgitation were present.

Adequate transmitral flow recordings were obtained in 106 patients on day 1, 104 patients on day 2, 96 patients on day 3,99 patients on day 7, 108 patients after three weeks, 102 patients after six weeks, 101 and 89 patients after three and six months, respectively, and 87 patients after one year.

According to early filling deceleration time on day 1, patients were divided into groups with short $(<150 \mathrm{~ms})$ and normal deceleration time $(\geqslant 150 \mathrm{~ms})$. We chose this value because it is considered to be the lower limit of normal for deceleration time, and also because it is very close to the value identified by receiver operating characteristic curve (ROC) analysis as the one predicting cardiac death with the greatest accuracy (152 ms) (fig 1). Importantly, ROC analysis of deceleration time measured on day $3(167 \mathrm{~ms})$ showed that the cut off point was some $15 \mathrm{~ms}$ longer than on day 1 . In addition, both sensitivity and specificity of deceleration time in predicting cardiac mortality decreased from $69.5 \%$ on day 1 to $57 \%$ on day 3 .

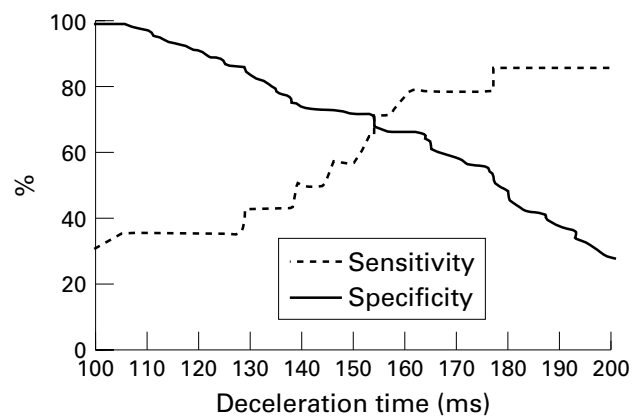

Figure 1 Receiver operating characteristic curve analysis to establish the most accurate value of deceleration time predictive of cardiac death.
Left ventricular end diastolic volume, end systolic volume, and ejection fraction were determined from apical two and four chamber views using Simpson's biplane formula. Tracing of endocardial borders in end diastole and end systole was performed on the Acuson 128 machine in the technically best cardiac cycle. The volumes were normalised for body surface area and expressed as indices. Left ventricular wall motion score index was calculated by using a 16 segment model. Wall motion for each segment was graded semiquantitatively as normokinesia $=1$, hypokinesia $=2$, akinesia $=3$, and dyskinesia $=4$. Wall motion score index was calculated by summing the scores for each segment and dividing by the number of analysed segments.

\section{INTEROBSERVER AND INTRAOBSERVER}

VARIABILITY

To test the reproducibility of the echocardiographic data, end diastolic and end systolic volume indices, ejection fraction, wall motion score index, and deceleration time were measured by two observers and twice by one observer, in 20 random patients. For two observers, coefficients of variation for measuring end diastolic and end systolic volume indices, ejection fraction, wall motion score index, and deceleration time were $6 \%, 9 \%, 9 \%, 3 \%$, and $5 \%$, respectively. For one observer, these values for repeating measurements were $4 \%$, $6 \%, 5 \%, 2 \%$, and $3 \%$, respectively.

\section{CORONARY ANGIOGRAPHY}

Coronary angiography was performed in 107 patients before hospital discharge, and deceleration time was measured in 94 of these. Stenosis of the major coronary artery of $\geqslant 70 \%$ was considered significant. Perfusion of the infarct related artery was assessed using TIMI (thrombolysis in myocardial infarction) criteria. $^{10}$

\section{STATISTICAL ANALYSIS}

All data are expressed as mean (SD). We used $\chi^{2}$ and $t$ tests for comparisons between the subgroups for categorical and continuous variables, respectively. Analysis of variance (ANOVA) with the Scheffé post hoc test was used to analyse repeated measures of left ventricular volumes, ejection fraction, and wall motion score index. A logistic regression model was used to identify predictors of left ventricular chamber stiffness early after myocardial infarction. An actuarial survival curve was constructed using the Kaplan-Meier method. ROC analysis was performed to establish the most accurate value of deceleration time for predicting cardiac death. Multivariate Cox model analysis was performed to assess the independent contribution of deceleration time to one year and five year mortality (a probability value of $\mathrm{p}<0.1$ was considered significant).

\section{Results}

\section{PATIENT CHARACTERISTICS}

On day 1, a short deceleration time was found in 32 of 106 patients (30\%). The percentage of patients with a short deceleration time 
Table 1 Baseline demographic, clinical, and angiographic characteristics of patients with and without short deceleration time on day 1 after myocardial infarction

\begin{tabular}{lll}
\hline & $\begin{array}{l}\text { Short DT } \\
(n=32)\end{array}$ & $\begin{array}{l}\text { Normal DT } \\
(n=74)\end{array}$ \\
\hline Age (years) & $52(7)$ & $55(8)$ \\
Sex (F/M) & $6 / 26$ & $18 / 56$ \\
Hypertension & $10 / 32$ & $31 / 74$ \\
Diabetes mellitus & $3 / 32$ & $13 / 74$ \\
Smoking & $20 / 32$ & $43 / 74$ \\
Killip class >1 & $5 / 32$ & $8 / 74$ \\
Anterior infarct & $18 / 32$ & $20 / 74 \dagger$ \\
Peak CK value & $1287(628)$ & $915(827)^{\star}$ \\
Patent IRA & $17 / 30$ & $34 / 64$ \\
Multivessel CAD & $14 / 30$ & $28 / 64$ \\
Collaterals & $11 / 30$ & $21 / 64$ \\
\hline
\end{tabular}

Values are mean $(\mathrm{SD})$ or $\mathrm{n}$.

${ }^{\star} \mathrm{p}<0.05 ; \mathrm{tp}<0.01 v$ short deceleration time. $\mathrm{CAD}$, coronary artery disease; $\mathrm{CK}$, creatine kinase; DT, early filling deceleration time; IRA, infarct related artery.

Table 2 Baseline characteristics of the patients in whom deceleration time was measured on day 1 and of those who were excluded from the analysis

\begin{tabular}{lll}
\hline & $\begin{array}{l}\text { DT measured } \\
(n=106)\end{array}$ & $\begin{array}{l}\text { DT not measured } \\
(n=25)\end{array}$ \\
\hline Age (years) & $54(9)$ & $58(9)^{\star}$ \\
Sex (F/M) & $24 / 82$ & $12 / 13^{\star}$ \\
Hypertension & $41 / 106$ & $9 / 25$ \\
Diabetes mellitus & $16 / 106$ & $3 / 25$ \\
Smoking & $63 / 106$ & $7 / 25 \dagger$ \\
Killip class >1 & $13 / 106$ & $16 / 25 \ddagger$ \\
Anterior infarct & $38 / 106$ & $16 / 25^{\star}$ \\
Peak CK value & $1021(787)$ & $1364(713)^{\star}$ \\
ESVi on day 1 (ml/m $\left.\mathrm{m}^{2}\right)$ & $32.02(10.29)$ & $43.70(15.72) \ddagger$ \\
EDVi on day 1 (ml/m $\left.\mathrm{m}^{2}\right)$ & $60.14(15.26)$ & $68.42(15.76) \dagger$ \\
EF on day 1 (\%) & $47.53(9.82)$ & $37.43(12.83) \ddagger$ \\
WMSi on day 1 & $1.53(0.30)$ & $1.87(0.42) \ddagger$ \\
Patent IRA & $51 / 94$ & $7 / 13$ \\
Multivessel CAD & $42 / 94$ & $9 / 13$ \\
Collaterals & $32 / 94$ & $6 / 13$ \\
Cardiac mortality & $14 / 106$ & $13 / 25 \ddagger$ \\
\hline
\end{tabular}

Values are mean (SD) or $\mathrm{n}$

${ }^{*} \mathrm{p}<0.05 ; \mathrm{tp}<0.01 ; \neq \mathrm{p}<0.001$

CAD, coronary artery disease; CK, creatine kinase; DT, early filling deceleration time; EDVi, end diastolic volume index; EF, ejection fraction; ESVi, end systolic volume index; IRA, infarct related artery; WMSi, wall motion score index.

decreased to $25 \%$ and $24 \%$ on day 3 and 7 , respectively, with a further decrease to $18 \%$ at 12 months. Baseline demographic, clinical, and angiographic characteristics of the patients with short and normal deceleration time on day 1 are shown in table 1 . Patients with a short deceleration time had larger infarcts, measured by peak creatine kinase value, and were more likely to have anterior infarcts.

When patients in whom deceleration time was measured on day 1 were compared with those who were excluded from the analysis because of sinus tachycardia, atrial fibrillation, or significant mitral regurgitation, it was found that the patients in the latter group were older, more often female, had larger infarcts, and had a higher incidence of anterior infarcts, Killip class $>1$, and diabetes mellitus (table 2). In addition, these patients had higher left ventricular volumes and wall motion score indices on day 1 and a lower ejection fraction. Finally, patients excluded from the measurement of deceleration time had a higher mortality during follow up.

ECHOCARDIOGRAPHIC FINDINGS

At baseline, both global and regional contractile function (ejection fraction and wall motion score index) were significantly better (49.5
$(9.7) \% \quad v \quad 44.0(9.7) \%, \mathrm{p}=0.04$ and 1.45 $(0.30) \%$ v $1.69(0.26) \%, \mathrm{p}=0.002$, respectively) in the group with normal deceleration time than in the group with a short deceleration time. During the follow up, according to ANOVA, a significant progressive improvement of ejection fraction was observed in patients with a normal deceleration time $(p=0.002)$, whereas no change was detected in patients with a short deceleration time $(\mathrm{p}=0.2)$. Additionally, a progressive decrease in wall motion score index was observed in the group with a normal deceleration time $(p<0.0001)$, while no change was observed in the group with a short deceleration time $(p=0.67)$. In contrast, comparisons between the groups by ANOVA showed that the pattern of changes in global and regional contractile function were similar $(p=0.91$ and $p=0.14$, respectively) (fig $2 \mathrm{~A}, \mathrm{~B}$ ).

Similarly, initial end diastolic and end systolic volume indices were higher in patients with a short deceleration time than in those with a normal deceleration time $(65.0$ (8.3) $v 57.2$ (10.2) $\mathrm{ml} / \mathrm{m}^{2}, \mathrm{p}=0.005$, and 36.9 (10.1) $v$ $29.3(9.4) \mathrm{ml} / \mathrm{m}^{2}, \mathrm{p}=0.005$, respectively). A progressive increase in end diastolic volume index was observed in both groups $(\mathrm{p}<0.001$, for both), whereas a progressive increase in end systolic volume index was observed only in patients with a short deceleration time $(\mathrm{p}=0.007)$, with no change in those with a normal deceleration time $(\mathrm{p}=0.58)$. Furthermore, comparisons between groups by ANOVA revealed that both end diastolic and end systolic volume indices showed a greater increase in the group with short deceleration time than in the group with normal deceleration time $(\mathrm{p}<0.0001$ and $\mathrm{p}=0.004$, respectively) (fig $2 \mathrm{C}, \mathrm{D}$ ).

When deceleration times in studies other than on day 1 were analysed, no consistent difference could be shown in left ventricular volumes, ejection fraction, and wall motion score index between groups with short and normal deceleration times during the one year follow up. Therefore only the initial deceleration time was used for further analysis.

PREDICTORS OF SHORT DECELERATION TIME ON DAY 1

A logistic regression model-which included infarct location, Killip class, peak creatine kinase value, initial left ventricular volume indices, ejection fraction and wall motion score index, extent of coronary artery disease, patency of the infarct related artery, and the presence of collaterals-identified initial wall motion score index as the only predictor of short deceleration time on day $1(\mathrm{p}=0.005)$.

PROGNOSTIC VALUE OF DECELERATION TIME AFTER MYOCARDIAL INFARCTION

A Cox model - which included infarct location, infarct size (measured by peak creatine kinase value), initial deceleration time, ejection fraction, and end systolic volume index-was constructed to assess this issue. A model was constructed for both one year and five year mortality. Because by definition end systolic 

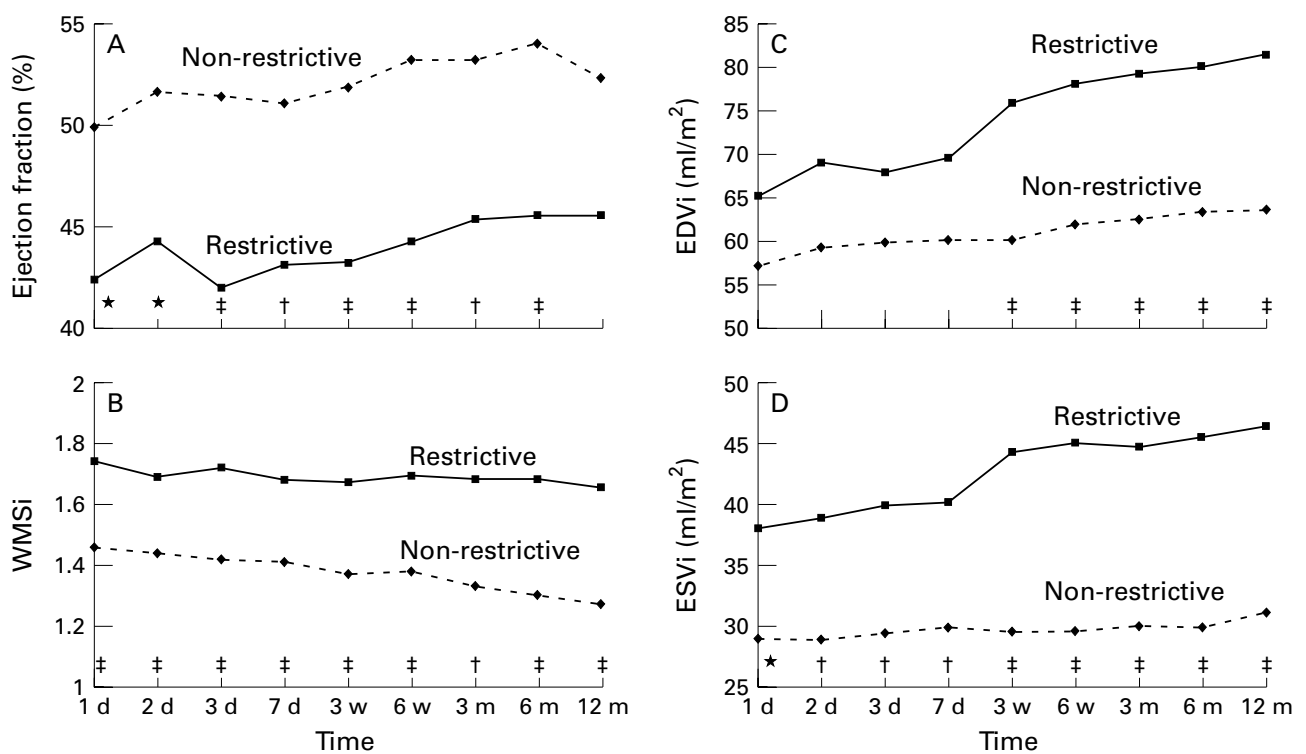

Figure 2 Changes in ejection fraction $(A)$, wall motion score index $(B)$, and end diastolic $(C)$ and end systolic volume index (D) during the follow up in patients with and without short deceleration time on day 1. EDVi, end diastolic volume index; ESVi, end systolic volume index; WMSi, wall motion score index. ${ }^{\star} p<0.05 ; \dagger p<0.01 ; \ddagger p<0.001$, between groups.

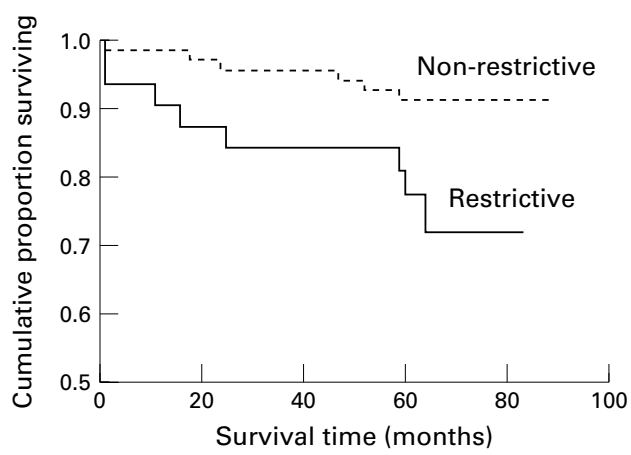

Figure 3 Actuarial survival curve in patients with and without short deceleration time on day $1(p=0.02)$.

volume index and ejection fraction are interrelated, they were not included simultaneously in the same model. We found that none of the examined variables was predictive of one year mortality. When initial ejection fraction was entered into the model, deceleration time on day 1 was the only variable predictive of five year mortality $(\beta=-1.1, p=0.05)$. On the other hand, when ejection fraction was substituted by initial end systolic volume index, this variable was identified as the only predictor of five year mortality $(\beta=1.7, \mathrm{p}=0.006)$.

FOLLOW UP

During follow up (59 (19) months, range 1-89 months), 14 of 106 patients with deceleration time measured on day 1 died of cardiac causes (eight of the 32 patients with a short deceleration time and six of the 74 patients with a normal deceleration time). During the first year after infarction, actuarial survival analysis showed higher cardiac mortality in patients with a short deceleration time (log-rank $\left.\chi^{2}=4.00, p=0.04\right)$; this trend was even more pronounced during the five year follow up (log-rank $\chi^{2}=5.34, \mathrm{p}=0.02$ ) (fig 3).

\section{Discussion}

DECELERATION TIME AND LEFT VENTRICULAR REMODELLING

Our study showed that a short initial deceleration time was associated with left ventricular remodelling during the first year after acute myocardial infarction. To our knowledge, this is the first study demonstrating that a single Doppler measurement, obtained as early as day 1 after infarction, can identify patients prone to short and long term ventricular dilatation. Recently, Cerisano and colleagues ${ }^{11}$ have reported, in a selected group of patients with reperfused acute anterior myocardial infarction, that a short deceleration time on day 3 is a strong predictor of left ventricular dilatation in the following six months. In our study, this observation was extended to unselected patients following infarction. We also showed that extensive left ventricular remodelling observed in these patients is associated with a worse prognosis.

An intriguing finding was that only deceleration time measured on day 1 could separate patients in whom left ventricular remodelling is likely to occur during the next year, while deceleration time measured in the later studies failed to do so. As clinical and experimental studies have shown that shortening of the deceleration time in the acute phase may be transient, ${ }^{12}{ }^{13}$ we hypothesised that deceleration time on day 1 may better reflect the functional damage caused by infarction than deceleration time in the later studies. In addition, owing to the selection bias inherent in analysing the prognostic value of repeatedly measured variables, the value of the initial variable has the greatest prognostic potential, as the sickest patients may die before the next measure is obtained. It should also be noted that the cut off point for most accurate value of deceleration time for predicting cardiac death changes over time, and the same values may not have identical implications at different time points. 
Finally, deceleration time values in later studies may be influenced by various therapeutic interventions during the acute and chronic phases of myocardial infarction.

In our study, the incidence of a short deceleration time decreased during follow up, supporting previous experimental findings ${ }^{13}$ that left ventricular diastolic function following myocardial infarction changes over time, probably because of a complex interplay of left ventricular dilatation, ischaemia, stunning, scar formation, and hypertrophy of non-infarcted segments. We have also recently shown that left ventricular chamber stiffness is increased 24-48 hours after infarction, but returned to normal within several days. ${ }^{12}$ Interestingly, it appears that changes in deceleration time are more pronounced in patients with an initially short deceleration time. ${ }^{11}$ These findings may be attributed, at least in part, to the decrease in left ventricular filling pressures caused by ventricular dilatation, and the subsequent prolongation of deceleration time. As ventricular dilatation is more pronounced in patients with an initially short deceleration time, it may be assumed that changes in filling pressures are greater, leading to pronounced changes in deceleration time over time in these patients.

We found that patients with a short deceleration time had larger infarcts, which is consistent with a previous finding that deceleration time is directly related to the size of the infarct. ${ }^{14}$ The only predictor of a short deceleration time was the initial wall motion score index, implying that the extent of infarction is the major determinant of early left ventricular chamber stiffness. As left ventricular dilatation is critically dependent on infarct size,${ }^{15}$ it appears that the amount of infarcted myocardium is the link between increased early chamber stiffness and subsequent left ventricular remodelling over the year following the infarct. These observations have been extended by Poulsen and colleagues, ${ }^{16}$ who have showed that a short deceleration time on day 1 predicts the development of congestive heart failure following acute myocardial infarction better than the ejection fraction. Thus the ability of a short deceleration time to identify patients prone to left ventricular dilatation as early as day 1 may have therapeutic and clinical implications in relation to attempts to prevent further remodelling. These patients might benefit from a more aggressive attempt to reopen the infarct related artery with alteplase ${ }^{17}$ or reteplase, ${ }^{18}$ or from primary percutaneous transluminal coronary angioplasty. ${ }^{19}{ }^{20}$ Also, acute administration of intravenous $\beta$ blockers ${ }^{21}$ and glyceryl trinitrate $^{22}$ to reduce infarct size, as well as the early use of ACE inhibitors ${ }^{23}$ to limit left ventricular remodelling, should be strongly recommended in these patients.

PROGNOSTIC VALUE OF DECELERATION TIME AFTER MYOCARDIAL INFARCTION

Survival analysis showed a significantly greater one year and five year cardiac mortality in patients with a short initial deceleration time. Although it has been shown that a restrictive filling pattern is associated with a dismal prognosis in patients with dilated cardiomyopathy ${ }^{24}{ }^{25}$ there are few data on the prognostic value of restrictive filling after myocardial infarction. While there is evidence that a short deceleration time can identify the patients with the worst prognosis, the prognostic value of deceleration time increments is less clear. It has recently been reported that restrictive filling is the single best predictor of cardiac mortality following myocardial infarction, and that it adds significantly to the predictive power of clinical and echocardiographic markers of systolic dysfunction. ${ }^{4}$ The Cox model analysis failed to identify any variable as an independent predictor of one year mortality, probably because of the relatively small number of patients and the low mortality during this period. Although independent predictors of five year mortality varied according to the model used, we believe that our findings support the value of deceleration time measurement for early prognostic assessment after acute myocardial infarction for the following reasons. First, deceleration time was found to be superior to ejection fraction. Second, deceleration time is easier to measure than left ventricular volumes and ejection fraction, making it appropriate for the use in acute settings. Third, in our series, deceleration time measurement was subjected to an almost twofold lower intraobserver and interobserver variability than end systolic volume index.

Exclusion criteria for deceleration time measurement used in our study were the same as those used by other groups, and all these criteria (sinus tachycardia, atrial fibrillation, and significant mitral regurgitation) have been shown to be predictors of adverse outcome following infarction. ${ }^{26}$ It appears that by applying these criteria we have excluded patients with the worse prognosis, and that patients in whom deceleration time was obtained represent the group that has a more favourable prognosis. However, it should be emphasised that although deceleration time measurement represents selection bias per se, a short initial deceleration time can still identify patients who are at increased risk for cardiac death and in whom short and long term left ventricular remodelling is likely to occur.

\section{STUDY LIMITATIONS}

It may be argued that a deceleration time of $150 \mathrm{~ms}$ is too loose a cut off point to separate patients into groups with short and normal deceleration time. Other groups have used different definitions of restrictive physiology, which may preclude direct comparisons between the studies. However, a common denominator for all these definitions is short deceleration time, suggesting that similar pathophysiology was involved in patients in the different studies, irrespective of the definition used.

The terms "increased chamber stiffness" and "short deceleration time" were used interchangeably, although the formula relating chamber stiffness and deceleration time has not been validated in humans. 
CONCLUSIONS

Our data indicate that a short deceleration time on day 1 after acute myocardial infarction can identify patients who are likely to undergo left ventricular remodelling in the following year. This is associated with a higher one year and five year cardiac mortality.

We would like to thank Dr Leonardo Bolognese for his thoughtful comments.

1 McKay RG, Pfeffer MA, Pasternak RC, et al. Left ventricular remodeling after myocardial infarction: a corollary to infarct expansion. Circulation 1986;74:693-702.

2 Popović AD, Nešković AN, Babić R, et al. Independent impact of thrombolytic therapy and vessel patency on left impact of thrombolytic therapy and vessel patency on left ventricular dilatation after myocardial infarction:

3 White HD, Norris RM, Brandt PWT, et al. Left ventricular end-systolic volume as the major determinant of survival end-systolic volume as the major determinant of survival after recovery

4 Nijland F, Kamp O, Karreman AJP, et al. Prognostic implications of restrictive left ventricular filling in acute myocardial infarction: a serial Doppler echocardiographic study. $\mathscr{f}$ Am Coll Cardiol 1997;30:1618-24.

5 Choong CY. Diastolic function: its principles and function. In: Weyman $\mathrm{AE}$, ed. Principles and practice of echocardiography. Philadelphia: Lea and Febiger;1994:721-80.

6 Little WC, Ohno M, Kitzman DW, et al. Determination of left ventricular chamber stiffness from the time for deceleration of early left ventricular filling Circulation 1995;92:1933-9.

7 Gianuzzi P, Imparato A, Temporelli PL, et al. Dopplerderived mitral deceleration time of early filling as a strong predictor of pulmonary capillary wedge pressure in postinpredictor of pulmonary capillary wedge pressure in postin-
farction patients with left ventricular systolic dysfunction. $\mathcal{f}$ farction patients with left ventricu

8 Forrester JS, Diamond G, Chatterjee K, et al. Medical therapy of acute myocardial infarction by application of hemodynamic subsets. N Engl F Med 1976;295:1356-62.

9 Gianuzzi P, Temporelli PL, Bosimini E, et al. Independen and incremental prognostic value of Doppler-derived mitral deceleration time of early filling in both symptomatic and asymptomatic patients with left ventricular dysfunction. F Am Coll Cardiol 1996;28:383-90.

10 The TIMI study group. The thrombolysis in myocardial infarction (TIMI) trial. Phase I findings. $N$ Engl ff Med 1985;312:932-6.

11 Cerisano G, Bolognese I, Carrabba N, et al. Dopplerderived mitral deceleration time: an early strong predictor of left ventricular remodeling after reperfused anterior acute myocardial infarction. Circulation 1999;99:224-9. 12 Popović AD, Nešković AN, Marinković J, et al. Serial assess-
ment of left ventricular chamber stiffness after acute myoment of left ventricular chamber stiffness after

13 Raya TE, Gay RG, Lancaster L, et al. Serial changes in left ventricular relaxation and chamber stiffness after large myocardial infarction in rats. Circulation 1988;77:1424-31.

14 Johannessen KA, Cerquira MD, Stratton JR. Influence of myocardial infarction size on radionuclide and Doppler echocardiographic measurements of diastolic function. $\mathrm{Am}$ 7 Cardiol 1990;65:692-7.

15 Kim CB, Braunwald E. Potential benefits of late reperfusion of infarcted myocardium. Circulation 1993;88:2426-36.

16 Poulsen SH, Jensen SE, Gøtzhe O, et al. Evaluation and prognostic significance of left ventricular diastolic function assessed by Doppler echocardiography in the early phase of a first acute myocardial infarction. Eur Heart 7 1997;18: 1882-9.

17 The GUSTO investigators. An international randomized trial comparing four thrombolytic strategies for acute myocardial infarction. N Engl f Med 1993;329:673-82.

18 Bode C, Smalling RW, Berg G, et al. Randomized comparison of coronary thrombolysis achieved with double-bolus son of coronary thrombolysis achieved with double-bolus
reteplase (recombinant plasminogen activator) and frontloaded, accelerated alteplase (recombinant tissue plasminogen activator) in patients with acute myocardial infarction: the RAPID II investigators. Circulation 1996;94: 891-8.

19 Zijlstra F, de Boer MJ, Hoorntje JC, et al. A comparison of mmediate coronary angioplasty with intravenous streptokinase in acute myocardial infarction. $N$ Engl f Med 1993;328:680-4.

20 Michels KB, Yusuf S. Does PTCA in acute myocardial infarction affect mortality and reinfarction rates? A quantitative overview (meta-analysis) of the randomized clinical trials. Circulation 1995;91:476-85.

21 Yusuf S, Peto R, Lewis J, et al. Beta blockade during and after myocardial infarction: an overview of the randomized trials. Prog Cardiovasc Dis 1985;27:335-71.

22 Jugdutt BI, Warnica JW. Intravenous nitroglycerin therapy to limit infarct size, expansion, and complications-effect of timing, dosage and infarct location. Circulation 1988;78: 906-19.

23 Pfeffer MA, Braunwald E, Moye LA, et al., on behalf of the SAVE investigators. Effect of captopril on mortality and morbidity in patients with left ventricular dysfunction after myocardial infarction: Results of the Survival and Ventricular Enlargement trial. N Engl f Med 1992;327:669-77.

24 Werner GS, Schaefer C, Dirks R, et al. Doppler echocardiographic assessment of left ventricular filling in idiopathic dilated cardiomyopathy during a one-year follow-up: relation to the clinical course of disease. Am Heart f 1993; 126:1408-16.

25 Rihal CS, Nishimura RA, Hatle LK, et al. Systolic and diastolic dysfunction in patients with clinical diagnosis of dilated cardiomyopathy: relation to symptoms and prognosis. Circulation 1994;90:2772-9.

26 Hochman JS, Gersh BJ. Acute myocardial infarction: complications. In: Topol ET, ed. Textbook of cardiovascular medicine. Philadelphia: Lipincot-Raven;1997:437-80.

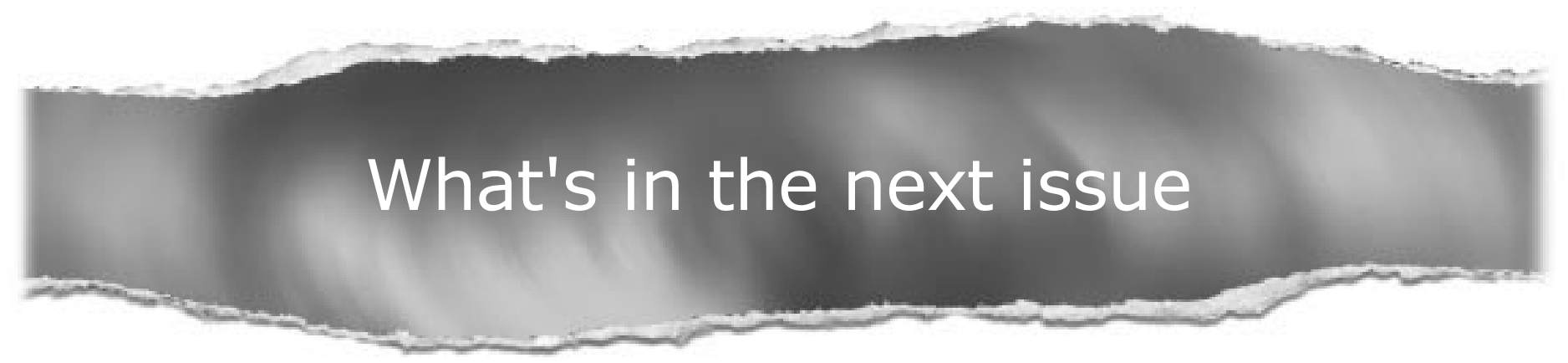

Future content

See which articles have just been accepted for publication and preview the table of contents for the next issue a month before it is published

\section{www.heartjnl.com}

